\title{
Factors associated with diverticular bleeding and re-bleeding: A United States hospital study
}

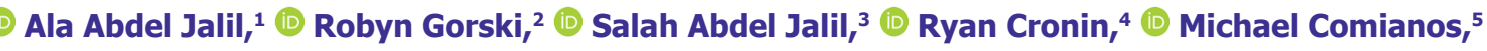 \\ (D) Moss Mann, ${ }^{6}$ (D) Hari Rajagopalan, ${ }^{7}$ (D) Asem Abdel Jalil, ${ }^{8}$ (D) Veysel Tahan ${ }^{1}$ \\ ${ }^{1}$ Department of Gastroenterology \& Hepatology, University of Missouri-Columbia, Columbia, Missouri, USA \\ 2Department of Anesthesiology, Medical College of Wisconsin, Milwaukee, Wisconsin, USA \\ 3Department of General Surgery, Grand Strand Medical Center, Myrtle Beach, South Carolina, USA \\ ${ }^{4}$ Department of General Surgery, East Tennessee State University, Johnson City, Tennessee, USA \\ ${ }^{5}$ Department of Internal Medicine, Ohio Health Riverside Methodist Hospital, Columbus, Ohio, USA \\ ${ }^{6}$ Department of Gastroenterology, Carolinas Medical Center, Florence, South Carolina, USA \\ ${ }^{7}$ School of Business, Francis Marion University, Florence, South Carolina, USA \\ ${ }^{8}$ Department of Pulmonary, Critical Care and Sleep Medicine, University of Missouri, Kansas, Missouri, USA
}

\begin{abstract}
OBJECTIVE: Diverticular bleeding is the most common cause of lower gastrointestinal bleeding. Arteriovascular disease, metabolic syndromes, non-steroidal anti-inflammatory drugs (NSAIDs), anti-thrombotics, and anticoagulants have been suggested as risk factors. There is a paucity of studies addressing factors associated with diverticular re-bleeding, especially in the United States. The aim of this study is to evaluate factors associated with colonic diverticular bleeding and re-bleeding in a US community-based hospital.
\end{abstract}

METHODS: We conducted a retrospective case-control study to analyze the factors associated with diverticular bleeding. Between January 2010 and July 2011, 93 patients were admitted to our hospital with a primary diagnosis of acute diverticular bleeding. We compared them to 152 patients who were admitted with a primary diagnosis of diverticulitis in the same period. We collected data from the medical records of each patient in relation to the demographics, comorbidities, medications, social habits, location of diverticulosis, length of stay in the hospital, and re-bleeding rate within 2 years of the first bleeding episode.

RESULTS: Factors such as cerebrovascular accident $(p=0.009)$, coronary artery disease $(p=0.037)$, diabetes mellitus $(p=0.046)$, obstructive sleep apnea $(p=0.033)$, NSAIDs $(p=0.038)$, use of anti-thrombotics $(p=0.001)$, anticoagulants $(p=0.002)$ or calcium channel blockers $(p=0.009)$, and bilateral diverticulosis $(p=0.001)$ were significantly associated with diverticular bleeding as compared to diverticulitis. Recurrence of bleeding was noted in 26 out of 93 patients (28\%) within 2 years of the first bleeding episode ( $p=0.001$ ). Bilateral colonic involvement, anticoagulants, and elderly age ( $\geq 65$ years) were found to have a closer relationship to diverticular re-bleeding, although it was not statistically significant.

CONCLUSION: This study reveals that arteriovascular disease, diabetes mellitus, NSAIDs, the use of anti-thrombotics, anticoagulants or calcium channel blockers, and obstructive sleep apnea are factors that are significantly associated with diverticular bleeding. It also shows that bilateral colonic involvement, elderly age, and anticoagulants have a closer relationship to diverticular re-bleeding. More prospective studies in patients with diverticular bleeding should be conducted to shed light on the causality of these factors and the prevalence of diverticulitis.

Keywords: Bleeding; community; diverticula; diverticulum; factor; outcome.

Cite this article as: Abdel-jalil A, Gorski R, Abdel Jalil S, Cronin R, Comianos M, Mann M, et al. Factors associated with diverticular bleeding and re-bleeding: A United States hospital study. North Clin Istanb 2019;6(3):248-253.

Received: December 19, 2017 Accepted: August 06, 2018 Online: September 05, 2018

Correspondence: Dr. Ala ABDEL JALIL. One Hospital Dr., CE 04165212 Columbia, United States.

Tel: +1573-884-6044 e-mail: ala79md@gmail.com

(c) Copyright 2019 by Istanbul Provincial Directorate of Health - Available online at www.northclinist.com 
$\mathrm{D}$ iverticular disease accounts for 312,000 admissions and 1.5 million days of inpatient care annually in the United States, with costs of care exceeding 2.6 billion dollars [1]. Diverticular disease of the gastrointestinal tract is more common in developed countries and occurs more often in older individuals. Prevalence of diverticular disease increases from $50 \%$ to $66 \%$ in patients older than 80 years [1], and is equally seen in both men and women [2]. Many patients diagnosed with diverticulosis remain asymptomatic, while approximately $10 \%-20 \%$ of patients eventually develop symptoms such as diverticular hemorrhage [3]. Although the patients with diverticulosis usually remain asymptomatic, symptomatic patients suffer from various symptoms ranging from irritable bowel syndrome (IBS)-type symptoms to disabling recurrent abdominal pain [4]. Diverticular hemorrhage can become severe with substantial morbidity and a mortality rate of $10 \%-20 \%[5]$.

The pathogenesis of diverticular bleeding is not completely understood but one common theory is that it originates over time because of repetitive injury to the vasa recta due to muscle contraction. Approximately 70\%$80 \%$ of diverticular bleedings resolve spontaneously, with re-bleeding occurring in up to $38 \%$ of patients [6]. Patients with uncomplicated diverticulitis generally respond well to outpatient conservative treatment, such as oral antibiotics. However, surgical treatment may be required for patients with recurrent diverticular bleeding and for diverticulitis complicated by a large abscess formation, perforation, intestinal obstruction, or fistula formation [7].

While some studies have assessed the factors associated with diverticular hemorrhage, there is a paucity of studies evaluating the factors associated with diverticulitis, diverticular bleeding, and diverticular rebleeding, most notably in the United States. Factors that have been postulated to be associated with an increased risk for diverticular bleeding include arteriovascular disease, metabolic syndrome, non-steroidal anti-inflammatory drugs (NSAIDS), anti-thrombotics, and anticoagulants. NSAIDs and aspirin have been identified to increase the risk for acute lower gastrointestinal bleeding, including acute diverticular bleeding and re-bleeding. Other factors associated with diverticular bleeding include a large number of diverticula, hypertension, and arteriosclerotic diseases including ischemic heart disease and chronic renal failure [8]. Patients with chronic kidney disease have been noted to display radiologic findings consistent with divertic- ular disease, yet remain asymptomatic [9]. The use of calcium channel blockers has also been suggested as a factor associated with a higher incidence of diverticular bleeding [10].

The purpose of this study was to evaluate the factors associated with colonic diverticular bleeding and re-bleeding (defined as a hospital admission within two years of a previous diverticular bleeding) in a United States hospital.

\section{MATERIALS AND METHODS}

In this study, we utilized a case-control approach to analyze factors associated with diverticular bleeding and re-bleeding. Between January 2010 and July 2011, 93 patients were admitted to our hospital with a primary diagnosis of acute diverticular bleeding based on clinical presentation and colonoscopic and radiographic examinations conducted during the hospital stay.

Diverticular bleeding was defined as lower gastrointestinal bleeding manifested by painless hematochezia in presence of active bleeding or stigmata of bleeding from affected diverticula during the colonoscopic exam, without the presence of another identifiable bleeding source. To analyze the factors associated with diverticular hemorrhage, a control group consisting of 152 patients was used. All the patients were admitted within the same time period with a primary diagnosis of acute diverticulitis based on clinical presentation and radiologic evidence of diverticulitis on computed tomography. We used diverticulitis as the control group since this condition is one of the major complications of diverticular disease and patients generally require hospital admission. The medical records of each patient in the case and control groups were screened for information regarding demographics, comorbidities, medications, findings of colonoscopy, location of diverticulosis, outcome of admission, complications including surgical intervention, hemoglobin level at time of presentation, and re-bleeding rate within 2 years of the first bleeding episode. We excluded patients who were pregnant, patients who had a concomitant severe active medical illness (including sepsis, myocardial infarction, or stroke), patients with end-stage renal disease or terminal cancer, and patients who were admitted for other medical or surgical reasons but developed gastrointestinal bleeding during their admission. Informed consent was obtained from all the participants. This study was approved by each institutional review board involved in the study. 
TABLE 1. Demographics and characteristics of patients and comparison of different variables between diverticulosis and diverticulitis groups

\begin{tabular}{|c|c|c|c|}
\hline & Diverticulosis (case group) & Diverticulitis (control group) & $\mathrm{p}$ \\
\hline Age (years), mean \pm SD & $73.3 \pm 12.1$ & $59.2 \pm 13.1$ & $<0.001$ \\
\hline Gender (M/F) & $1.4: 1$ & $1.8: 1$ & 0.37 \\
\hline Race (W/B) & $1: 1$ & $1.7: 1$ & $0.035^{*}$ \\
\hline Length of stay (days) & $5.85 \pm 1.41$ & $6.11 \pm 0.71$ & $>0.05$ \\
\hline Hemoglobin level at admission $(\mathrm{g} / \mathrm{dL})$, mean \pm SD & $10.2 \pm 2.46$ & $12.6 \pm 2.0$ & $<0.001$ \\
\hline $\mathrm{BMI}$, mean $\pm \mathrm{SD}$ & $32.2 \pm 5.0$ & $31.2 \pm 10.8$ & 0.068 \\
\hline Hypertension, \% & 74.1 & 75 & 0.88 \\
\hline Cerebrovascular accident, \% & 13.9 & 4.6 & 0.009 \\
\hline Coronary artery disease, $\%$ & 32.2 & 20.3 & 0.04 \\
\hline Diabetes mellitus, \% & 35.4 & 23.6 & 0.046 \\
\hline Chronic kidney disease, $\%$ & 23.6 & 15.7 & 0.12 \\
\hline Obstructive sleep apnea, \% & 7.5 & 1.9 & 0.03 \\
\hline Gout, \% & 11.8 & 8.5 & 0.40 \\
\hline Alcohol use, \% & 12.9 & 7.2 & 0.14 \\
\hline Smoking, \% & 15 & 18.4 & 0.49 \\
\hline NSAIDs use, $\%$ & 18.2 & 9.2 & 0.03 \\
\hline Aspirin/anti-thrombotics, \% & 48.3 & 23.6 & 0.001 \\
\hline Warfarin/anticoagulants, \% & 15.0 & 2.6 & 0.002 \\
\hline Proton pump inhibitor, $\%$ & 25.8 & 32.2 & 0.28 \\
\hline ACEI/ ARB, \% & 49.4 & 49.3 & 0.98 \\
\hline Beta blocker, \% & 46.2 & 34.2 & 0.06 \\
\hline Calcium channel blockers, \% & 37.6 & 22.3 & 0.01 \\
\hline
\end{tabular}

*Was more significant in black patients. BMI: Body mass index; NSAIDs: Non-steroidal anti-inflammatory drugs; ACEI: Angiotensin-converting enzyme inhibitor; ARB: Angiotensin receptor blocker.

\section{Statistical Analysis}

Descriptive methods utilizing mean and standard deviation were employed. To examine the differences in the distribution among case and control groups, the Student's t-test, Pearson Chi-Square, or Fischer's Exact Test were used when appropriate. Logistic regression analysis was used to examine the interaction between different factors. An alpha level of significance was set at $<0.05$.

\section{RESULTS}

Forty-three (46\%) patients included in the case group had diverticular disease in the left colon, $5(5 \%)$ in the right, and $37(40 \%)$ presented with bilateral diverticular disease. Eight patients (9\%) did not have a colonoscopy for various reasons, including advanced age. In the control group, $94 \%$ of the participants had diverticular disease in the left colon. The mean age for case and control groups was $73.3 \pm 12.1$ years and $59.2 \pm 13.1$ years, respectively. There was no significant difference between the two groups with regard to gender, body mass index (BMI) (32.2 \pm 5.0 and $31.2 \pm 10.8$, respectively), or length of stay $(5.85 \pm 1.41$ days and 6.11 \pm 0.71 days, respectively). African American patients were found to have a higher association with diverticular bleeding as compared to diverticulitis (white: black ratio $=1: 1$ and $1.7: 1$, respectively for diverticular bleeding and diverticulitis; $p=0.035$ ). Table 1 shows the demographics and characteristics of patients and the comparison of different variables between the case and control groups.

Hypertension was not found to be significantly associated with either diverticular bleeding or re-bleeding, whereas cerebrovascular accident (CVA) was found to be significantly associated with diverticular bleeding $(p=0.009)$ but not re-bleeding. This pattern of asso- 
ciation with diverticular bleeding but not re-bleeding was also seen with coronary artery disease (CAD) $(p=0.037)$, diabetes mellitus $(p=0.046)$, and obstructive sleep apnea (OSA) $(p=0.033)$. Chronic kidney disease (defined as GFR $<60 \mathrm{ml} / \mathrm{min} / 1.73 \mathrm{~m}^{2}$ for $>3$ months) was not shown to be significantly associated with diverticular bleeding or re-bleeding. Multiple logistic regression analysis showed no significant interaction between these variables that indicated an increase in the risk of diverticular bleeding or re-bleeding.

Diverticular bleeding, but not re-bleeding, was shown to increase in patients who used NSAIDs or aspirin and calcium channel blockers (CCBs). In contrast, proton pump inhibitors (PPIs) showed no difference in relation to diverticular bleeding or re-bleeding. In patients taking both NSAIDs and a PPI, no interaction effect was seen with respect to an increase in the risk of bleeding or re-bleeding. Anticoagulants such as warfarin and anti-thrombotic drugs such as aspirin and Plavix showed a higher association with diverticular bleeding, however, they were not statistically significant for diverticular re-bleeding $(\mathrm{p}=0.11$ for anticoagulant use; $p=0.5$ for aspirin/anti-thrombotic use). Alcohol use (defined as $>2$ drinks/day for males and $>1$ drink/ day for females) among the case group was not found to be significantly associated with either diverticular bleeding or re-bleeding as compared to the diverticulitis group.

Gender differences were not significant between groups with respect to the rates of bleeding and re-bleeding. In our study, patients were divided into four groups on the basis of age: very elderly ( $\geq 80$ years), elderly (6579 years), middle age (45-64 years), and young ( $\leq 44$ years). The older a patient, the higher the association with diverticular bleeding; we found that 27 of 35 (77\%) very elderly patients, 39 of 81 (48\%) of elderly patients, 26 of 105 (25\%) middle-aged patients, and only 4\% of young patients in the case group developed diverticular hemorrhage. The very elderly patients were the closest of the four groups to being statistically significant for rebleeding $(\mathrm{p}=0.08)$. Patients were grouped based on BMI as: normal (BMI 18-25), overweight (BMI 25-29.9), and obese $(B M I \geq 30)$; this factor was not significantly different between the two groups for either bleeding or re-bleeding.

Left-sided bleeding occurred more commonly out of the three groups and right-sided bleeding occurred least commonly. Bilateral diverticulosis was signifi- cantly associated with diverticular bleeding $(p=0.001)$. Recurrence of bleeding was noted in 26 of 93 (28\%) case group patients within 2 years of the first bleeding episode $(\mathrm{p}=0.001)$.

While there are a myriad of outcomes for patients presenting to the hospital with diverticular bleeding, death as an outcome was not shown to be more prevalent among those patients hospitalized with diverticular bleeding versus diverticulitis. The length of stay in the hospital and the risk for re-bleeding were independent of each other in this study; no significant correlation was found. As was expected, at the time of admission, the patients presenting with diverticular bleeding had significantly lower levels of hemoglobin than those with diverticulitis $(\mathrm{p} \leq 0.01)$.

\section{DISCUSSION}

Diverticular bleeding accounts for many hospitalizations throughout the world, yet few studies have identified specific factors leading to an increased risk of bleeding. Our study confirms that certain comorbidities are more associated with diverticular bleeding, including cardiovascular diseases (CVA, CAD) and diabetes mellitus. The association between OSA and diverticular hemorrhage is the first of its kind to be mentioned in the literature. Such an association could be explained by the higher prevalence of sleep apnea among patients with cardiovascular disease, who in turn have a higher risk for diverticular hemorrhage. A previous study noted that apart from NSAIDs and anticoagulants, arterial hypertension had been shown to be an independent risk factor for colonic diverticular bleeding [11]. This indicates that arteriosclerosis and associated diseases such as metabolic syndrome could, therefore, play an important role in the pathogenesis of acute diverticular bleeding. However, our study did not show that hypertension is associated with a higher risk for diverticular bleeding. The high prevalence of hypertension and metabolic syndrome in our study population might be the reason for this nonsignificant association.

In our study, the African American and elderly patients were shown to have a higher association with diverticular bleeding, while gender had no bearing on the risks of bleeding or re-bleeding, as has been previously stated in the literature. Although approximately $70 \%-$ $80 \%$ of diverticular bleeding resolves spontaneously, rebleeding occurs in up to $38 \%$ of patients, thus making it a pertinent factor to explore. In our study, we found a very 
TABLE 2. Factors with close significance for diverticular rebleeding

\begin{tabular}{lc} 
& $\mathrm{p}$ \\
\hline Bilateral colonic involvement & 0.10 \\
Anticoagulant use & 0.11 \\
Very elderly age $(\geq 80)$ & 0.08
\end{tabular}

elderly age $(\geq 80)$ to be an important factor for re-bleeding, close to reaching statistical significance $(p=0.08)$. The location of the bleed was also examined and bilateral disease was a significant factor for diverticular bleeding, (bilateral: 9/39, left/sigmoid: 23/186, and right-sided disease: $2 / 12$ in both groups). Bilateral involvement was close to reaching statistical significance for diverticular re-bleeding $(\mathrm{p}=0.10)$. Table 2 shows the factors with close significance for diverticular re-bleeding.

Table 3 reveals a staggering difference in rates of rebleeding within 2 years of the first bleeding episode between the patients with diverticulosis versus those with diverticulitis, with the former resulting in significantly more re-bleeding incidences $(27.9 \%$ vs. $7.2 \%)$.

While the difference in death as an outcome between the two groups was shown to be insignificant, surgical intervention and complications (abscess or fistula formation, intestinal obstruction, peritonitis, sepsis, perforation, or the need for colonic resection or laparotomy) were higher in the diverticulitis group $(p<0.001)$.

Our study verifies that certain medications, including NSAIDs, aspirin, anticoagulants, and CCBs are associated with a higher rate of diverticular hemorrhage. The strong association between diverticular bleeding and the use of NSAIDs as evaluated by our study agrees with the current literature, however, unlike a previous study on diverticular bleeding in Japan [12] our study showed no increased risk of diverticular re-bleeding after NSAID use. This was also true for aspirin with respect to the risk of diverticular bleeding and re-bleeding. Anticoagulants such as warfarin and other anti-thrombotic drugs such as clopidogrel have been mentioned in the literature to increase the risk of diverticular bleeding, which our results concur with. On delving further into the effect of anticoagulants on re-bleeding, no association was found between any of the drugs and diverticular re-bleeding. The use of PPIs exhibited no increased diverticular bleeding or
TABLE 3. Outcome of admission, surgical intervention, complications, and re-bleeding rate

\begin{tabular}{lccc} 
& $\begin{array}{c}\text { Diverticulosis } \\
\text { (case group) } \\
\%\end{array}$ & $\begin{array}{c}\text { Diverticulitis } \\
\text { (control group) } \\
\%\end{array}$ & $\mathrm{p}$ \\
\hline $\begin{array}{l}\text { Outcome (death) } \\
\text { Surgical intervention }\end{array}$ & 3.2 & 1.3 & 0.3 \\
$\begin{array}{l}\text { Complications** } \\
\text { Re-bleeding }\end{array}$ & 5.3 & 26 & $<0.001^{*}$ \\
within 2 years & 10.7 & 33.5 & $<0.001^{*}$ \\
\hline
\end{tabular}

*Was more significant in the diverticulitis group. **Complications include abscess or fistula formation, intestinal obstruction, peritonitis, sepsis, perforation or need for colonic resection or laparotomy.

re-bleeding risk among patients. Our study also verifies that CCBs are associated with diverticular bleeding, an observation that has been described in a European study, but up to this point had yet to reveal itself in a US-based study.

This study used a retrospective case-control approach to identify factors associated with diverticular hemorrhage and delineate the common and distinctive factors associated with diverticular bleeding and re-bleeding. This approach may have led to selection bias. To address this issue, we selected patients who were admitted with a primary diagnosis of diverticulitis as the control group (contrary to other matching groups) since non-bleeding diverticulosis patients may not necessarily get admitted to the hospital. Other limitations include the possibility of the patients getting treated at other facilities for their illness (diverticular bleeding or re-bleeding), which may have led to an underestimation of the prevalence of rebleeding. Reviewing the patient population and demographics showed that a majority of the patients received their medical care at our facility, as it is the main hospital in the region. Our findings may show some overestimation due to higher prevalence of metabolic syndrome in our region.

\section{Conclusion}

This case-control study reveals that cardiovascular disease, diabetes mellitus, NSAIDs, anti-thrombotics, anticoagulants, CCBs, and OSA are significant risk factors associated with diverticular bleeding. It also suggests that bilateral colonic involvement increases the risk for diver- 
ticular bleeding. Bilateral involvement, anticoagulant use, and elderly age ( $\geq 65$ years) were factors that had a closer relationship to diverticular re-bleeding, although it was not statistically significant. This is the first study to introduce OSA as a possible risk factor associated with diverticular bleeding, which may have been mediated by the high prevalence of cardiovascular disease and metabolic syndromes in the study population. Advanced age, especially in very elderly patients ( $\geq 80$ years old) had a strong association with diverticular bleeding. CCBs have been described in a German study as a risk factor associated with diverticular hemorrhage; our study confirms this observation in a United States hospital. Interestingly, obesity was not shown to be a significant risk factor for diverticular bleeding in our study. This may be due to the high prevalence of obesity in the region where the study was conducted.

As a significant contributor to hospital admissions, diverticular bleeding, and re-bleeding, risk factors are prime targets for investigations in order to prevent or decrease the number of hospital admissions and to improve morbidity from the disease. Further investigation into the causality of these risk factors for diverticular bleeding and re-bleeding through prospectively designed studies is required.

Ethics Committee Approval: Study was approved by McLeod Regional Medical Center's IRB \& Ethics committee on May $16^{\text {th }}, 2013$.

Acknowledgement: The authors acknowledge Dr. Hari Rajagopalan from the School of Business at Francis Marion University in Florence, South Carolina for the valuable statistical help he provided for the study.

Conflict of Interest: The authors declare no conflict of interest.

Financial Disclosure: The authors declared that this study has received no financial support.
Authorship Contributions: Concept - AAJ, MM, RG; Supervision $A A J, V T, M M, H R$; Materials - AAJ, RC, MC, RG, SAJ; Data collection and/or processing - RG, RC, MC, SAJ; Analysis and/or interpretation - AAJ, MM, HR, AAJ, VT; Writing - RG, AAJ, SAJ; Critical review $A A J, V T, H R, A A J$.

\section{REFERENCES}

1. Matrana MR, Margolin DA. Epidemiology and pathophysiology of diverticular disease. Clin Colon Rectal Surg 2009;22:141-6. [CrossRef]

2. McConnell EJ, Tessier DJ, Wolff BG. Population-based incidence of complicated diverticular disease of the sigmoid colonbased on gender and age. Dis Colon Rectum 2003;46:1110-4. [CrossRef]

3. Stollman NH, Raskin JB. Diverticular disease of the colon. J Clin Gastroenterol 1999;29:241-52. [CrossRef]

4. Lee KM, Paik CN, Chung WC, Jung SH, Chang UI, Yang JM. Clinical significance of colonic diverticulosis associated with bowel symptoms and colon polyp. J Korean Med Sci 2010;25:1323-9. [CrossRef]

5. Chait MM. Lower gastrointestinal bleeding in the elderly. World J Gastrointest Endosc 2010;2:147-54. [CrossRef]

6. Raphaeli T, Menon R. Current treatment of lower gastrointestinal hemorrhage. Clin Colon Rectal Surg 2012;25:219-27. [CrossRef]

7. Oh HK, Han EC, Ha HK, Choe EK, Moon SH, Ryoo SB, et al. Surgical management of colonic diverticular disease: discrepancy between rightand left-sided diseases. World J Gastroenterol 2014;20:10115-20.

8. Niikura R, Nagata N, Akiyama J, Shimbo T, Uemura N. Hypertension and concomitant arteriosclerotic diseases are risk factors for colonicdiverticular bleeding: a case-control study. Int J Colorectal Dis 2012;27:1137-43. [CrossRef]

9. Galbraith P, Bagg MN, Schabel SI, Rajagopalan PR. Diverticular complications of renal failure. Gastrointest Radiol 1990;15:259-62.

10. Kaplan RC, Heckbert SR, Koepsell TD, Rosendaal FR, Psaty BM. Use of calcium channel blockers and risk of hospitalized gastrointestinal tract bleeding. Arch Intern Med 2000;160:1849-55. [CrossRef]

11. Yamada A, Sugimoto T, Kondo S, Ohta M, Watabe H, Maeda S, et al. Assessment of the risk factors for colonic diverticular hemorrhage. Dis Colon Rectum 2008;51:116-20. [CrossRef]

12. Tsuruoka N, Iwakiri R, Hara M, Shirahama N, Sakata Y, Miyahara K, et al. NSAIDs are a significant risk factor for colonic diverticular hemorrhage in elder patients: evaluation by a case-control study. J Gastroenterol Hepatol 2011;26:1047-52. [CrossRef] 